\title{
ARTICLE
}

Clinical Research

\section{Comparison of cardiovascular risk factors between children and adolescents with classes III and IV obesity: findings from the APV cohort}

\author{
Thomas Reinehr $\mathbb{D}^{1} \cdot$ Sascha R. Tittel $\mathbb{D}^{2} \cdot$ Rolf Holle $\mathbb{D}^{3} \cdot$ Susanna Wiegand ${ }^{4} \cdot$ Ines Gellhaus ${ }^{5}$. \\ Johannes Hebebrand ${ }^{6} \cdot$ Susanne Greber-Platzer $\mathbb{D}^{7} \cdot$ Christian Denzer $^{8} \cdot$ Sabine Linke $^{9} \cdot$ Wieland Kiess $^{10} \cdot$ \\ Reinhard W. Holl (iD ${ }^{2}$
}

Received: 17 April 2020 / Revised: 5 January 2021 / Accepted: 21 January 2021 / Published online: 7 April 2021

(c) The Author(s) 2021. This article is published with open access

\begin{abstract}
Objective Obesity is associated with many cardiovascular risk factors (CVRF) in childhood. There is an ongoing discussion whether there is a linear relationship between degree of overweight and deterioration of CVRFs justifying body mass index (BMI) cut-offs for treatment decisions.

Methods We studied the impact of BMI-SDS on blood pressure, lipids, and glucose metabolism in 76,660 children (aged 5-25 years) subdivided in five groups: overweight (BMI-SDS 1.3 to $<1.8$ ), obesity class I (BMI-SDS 1.8 to $<2.3$ ), class II (BMI-SDS 2.3-2.8), class III (BMI-SDS > 2.8-3.3), and class IV (BMI-SDS > 3.3). Analyses were stratified by age and sex. Results We found a relationship between BMI-SDS and blood pressure, triglycerides, HDL cholesterol, liver enzymes, and the triglycerides-HDL-cholesterol ratio at any age and sex. Many of these associations lost significance when comparing children with obesity classes III and IV: In females $<14$ years and males $<12$ years triglycerides and glucose parameters did not differ significantly between classes IV and III obesity. Prevalence of dyslipidemia was significantly higher in class IV compared to class III obesity only in females $\geq 14$ years and males $\geq 12$ years but not in younger children. In girls $<14$ years and in boys of any age, the prevalences of type 2 diabetes mellitus did not differ between classes III and IV obesity.

Conclusions Since a BMI above the highest BMI cut-off was not associated consistently with dyslipidemia and disturbed glucose metabolism in every age group both in boys and girls, measurements of CVRFs instead of BMI cut-off seem preferable to guide different treatment approaches in obesity such as medications or bariatric surgery.
\end{abstract}

Supplementary information The online version contains supplementary material available at https://doi.org/10.1038/s41366021-00773-x.

Thomas Reinehr

T.Reinehr@kinderklinik-datteln.de

1 Department of Pediatric Endocrinology, Diabetes and Nutrition Medicine, Vestische Hospital for Children and Adolescents Datteln, University of Witten/Herdecke, Witten, Germany

2 German Center for Diabetes Research, Institute of Epidemiology and Medical Biometry, University of Ulm, Ulm, Germany

3 Institute for Medical Informatics, Biometry and Epidemiology, University Hospital, Ludwig-Maximilians-University Munich, Munich, Germany

4 Charité-Universitätsmedizin Berlin, corporate member of Freie Universität Berlin, Humboldt-Universität zu Berlin, and Berlin Institute of Health, Berlin, Germany

5 Consensus Group Obesity Training for Children and Young Adults, Rehabilitation Clinic for Children and Adolescents, Sylt, Germany
6 Department of Child and Adolescent Psychiatry, Psychosomatics and Psychotherapy, University Hospital Essen, University of Duisburg-Essen, Essen, Germany

7 Comprehensive Center Pediatrics, Department of Pediatrics and Adolescent Medicine, Division of Pediatric Pulmonology, Allergology and Endocrinology, Medical University Vienna, Vienna, Austria

8 Department of Pediatrics and Adolescent Medicine, Division of Pediatric Endocrinology and Diabetes, University Medical Center Ulm, Ulm, Germany

9 Katholisches Kinderkrankenhaus WILHELMSTIFT gGmbH, Children's Hospital, Hamburg, Germany

10 University of Leipzig, Hospital for Children and Adolescents, Leipzig, Germany 


\section{Introduction}

Obesity is associated with many cardiovascular risk factors (CVRF) such as hypertension, dyslipidemia, and impaired glucose metabolism in childhood [1,2]. These CVRFs are expected to lead to premature death later in life. It has been demonstrated in a large longitudinal study over 40 years that mortality due to cardiovascular events rose with increase of body mass index (BMI) in adolescents [3]. Many studies reported a significant relationship between degree of overweight and CVRFs in children and adolescents [4-8]. This relationship is mediated by sex and age since CVRFs deteriorated during puberty and improved at the end of puberty [9].

There is an ongoing discussion whether a linear doseresponse relationship of increasing degree of overweight on the presence of CVRFs [3] plateaus above a certain threshold. It has been demonstrated in one previous study that children and adolescents with class III obesity as defined by $140-160 \%$ of the 95 th percentile of their BMI did not differ in their CVRFs from children and adolescents with class IV obesity as defined by $>160 \%$ of the 95th percentile of their BMI [10]. This finding may have practical relevance, because indications for different treatment approaches for obesity such as medications or bariatric surgery are usually based on defined cut-off points of degree of overweight $[11,12]$. However, large confirmatory studies are still lacking.

Therefore, we studied the impact of degree of overweight on CVRFs in a large cohort of children and adolescents with special focus on classes III and IV obesity subgroups using different classifications of obesity. By stratifying the analyses by sex and age groups, we tried to identify possible influencing factors on this relationship. We hypothesize according to the study of Zabarsky et al. [10] that children and adolescents with class IV obesity do not differ in their CVRFs from children and adolescents with class III obesity.

\section{Subjects and methods}

Based on the German guidelines for diagnostics and treatment of overweight children and adolescents [13], a computer software based on the visual foxpro 9.0 compiler was developed for standardized prospective documentation of overweight children and adolescents (APV) in 1999 (www.ap-v.de) [14]. Participation in this quality control program is the precondition for accreditation of obesity treatment centers by the German Obesity Society (DAG) and for funding of the treatment by health insurances. Anthropometric parameters, metabolic control, and treatment (modality and intensity of lifestyle interventions) are documented longitudinally by the software. The software allows standardized patient reports, local aggregation of data, and patient selection according to multiple criteria. Anonymized data are transmitted for central analysis. Each participating center complies with its local ethical and data management guidelines. Inconsistent data are reported back to the centers twice a year for correction.

A total of 224 centers specialized in pediatric obesity care in Germany, Austria, and Switzerland participated in this quality assessment. All patients at the age of $\geq 5$ years and up to 25 years presenting at these institutions with a BMI $>25 \mathrm{~kg} / \mathrm{m}^{2}$ or BMI-SDS $>1.3$ in the years 2006-2016 were included in the analysis. Children with syndromes were excluded. In the longitudinal analyses, children and adolescents with bariatric surgery and children and adolescents with long-term inpatient treatment $>6$ months were also excluded.

German population-based reference data for BMI were used as recommended by the International Task Force of Obesity (IOTF) [15]. The degree of overweight was quantified using Cole's Box Cox-transformation, which normalizes the BMI skewed distribution in childhood and expresses BMI as a standard deviation score (SDS-BMI) [16]. Degree of overweight was categorized in five classes based on two different approaches: (a) based on BMI-SDS as suggested by IOTF [overweight: BMI-SDS 1.3 to $<1.8$, class I obesity (mildly obese): BMI-SDS 1.8 to $<2.3$ corresponding to 97 th to $<99.5$ th BMI percentile, class II obesity (moderately obese): BMI-SDS 2.3-2.8 corresponding to 99.5 th to $<99.9$ th BMI percentile, class III obesity (extremely obese): BMI-SDS $>2.8-3.3$ [15], class IV obesity (super obese): BMI-SDS >3.3] and (b) as suggested by Zabarsky et al. [10] overweight as 85th-95th percentile of BMI and obesity according to the 95th percentile of BMI (obesity class I: 100-120\% of 95th percentile of BMI, class II: $>120-140 \%$ of the 95 th percentile of BMI, class III: $>140-160 \%$ of the 95 th percentile of BMI, class IV: $>160 \%$ of the 95 th percentile of BMI) [10]. Class I obesity corresponds to a BMI $30-35 \mathrm{~kg} / \mathrm{m}^{2}$ in adults; class II to a BMI $35-40 \mathrm{~kg} / \mathrm{m}^{2}$ in adults and class III to a BMI $>40 \mathrm{~kg} / \mathrm{m}^{2}$ in adults.

To account for a potential impact of puberty on CVRFs, we divided our study population in three classes for boys and girls, respectively: boys aged $<12$ years assuming prepuberty, boys aged 12 to $<16$ years assuming puberty, and boys aged $\geq 16$ years assuming postpuberty; girls aged $<10$ years assuming prepuberty, girls aged 10 to $<14$ years assuming puberty, and girls aged $\geq 14$ years assuming postpuberty.

To adjust for differences among different laboratory methods, the multiple of the mean method was applied to mathematically standardize $\mathrm{HbAlc}$ measurements to the Diabetes Control and Complications Trial reference range (4.05-6.05\%; 20.7-42.6 mmol/mol) [17]. An oral glucose tolerance test (oGTT) was performed according to recent international guidelines [18]. 


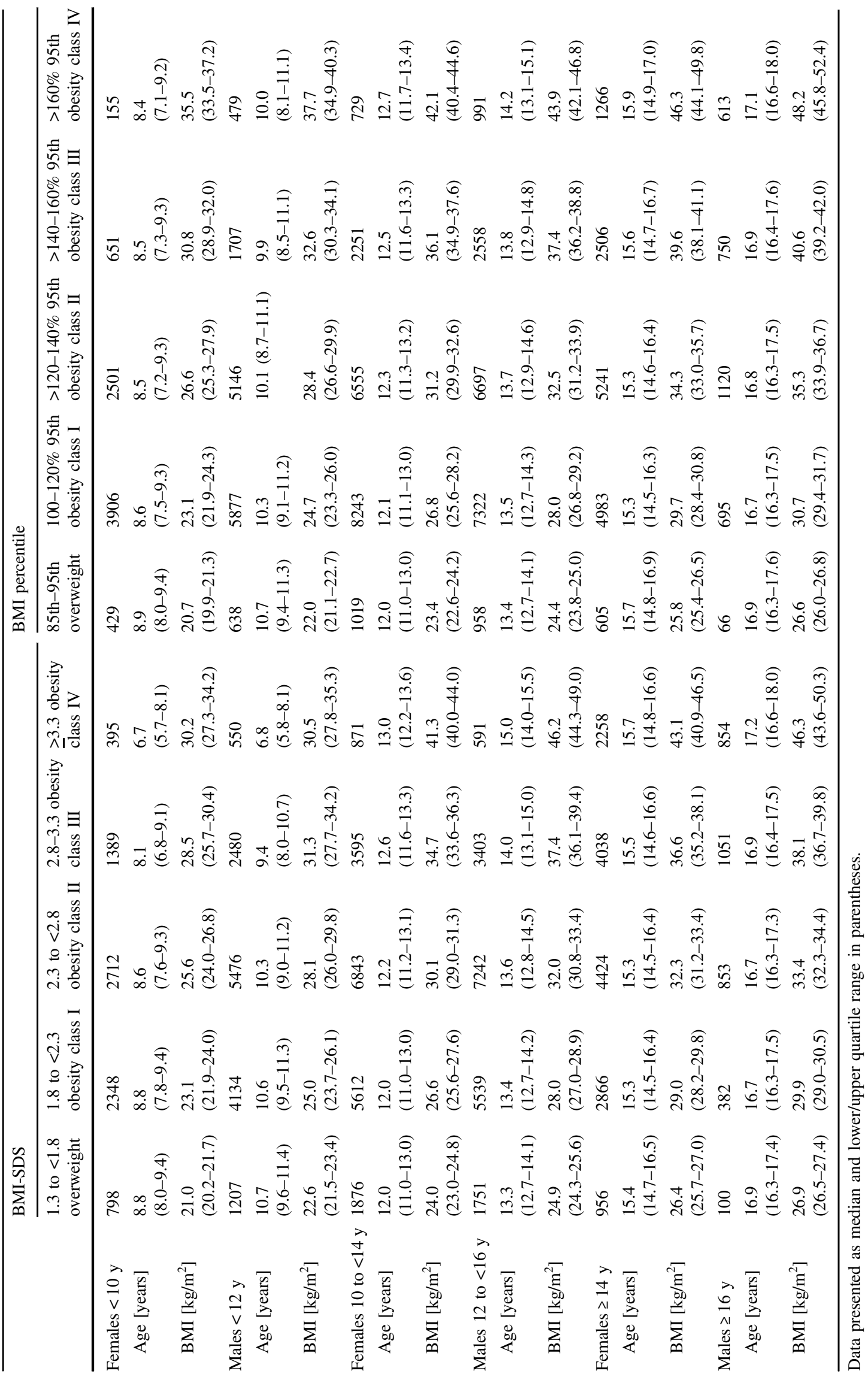


Non-HDL cholesterol was calculated by subtracting HDL cholesterol from total cholesterol. Dyslipidemia was defined by total cholesterol levels $>200 \mathrm{mg} / \mathrm{dl}$ or $\mathrm{HDL}<35 \mathrm{mg} / \mathrm{dl}$ or LDL $>130 \mathrm{mg} / \mathrm{dl}$ or triglycerides $>150 \mathrm{mg} / \mathrm{dl}$ according to German guidelines [19]. The triglycerides to HDLcholesterol ratio $(\mathrm{T} / \mathrm{H})$ was used as an indirect measurement of insulin resistance. Arterial hypertension was defined by blood pressure above the 95th percentile or $>140$ / $90 \mathrm{mmHg}$ using German percentiles [20,21] and diabetes by fasting glucose $\geq 126 \mathrm{mg} / \mathrm{dl}$ or 2 -h glucose in oGTT $\geq$ $200 \mathrm{mg} / \mathrm{dl}$ or $\mathrm{HbA} 1 \mathrm{c}>6.5 \%$ [18].

The CVRFs, transaminases (aspartate aminotransferase (AST), alanine aminotransferase (ALT), glutamate-pyruvate transaminase $(\gamma \mathrm{GT}))$, and the prevalence of hypertension, dyslipidemia, and type 2 diabetes mellitus (T2DM) were compared between the patients with classes III and IV obesity. Analyses were performed stratified by sex and age.

\section{Statistics}

Statistical evaluation was performed by SAS-version 9.4 (SAS Inst. Inc., Cary, NC, USA). Data are presented as median values and interquartile range. The relationship between BMI-SDS and CVRFs was calculated by linear regression models. Group comparisons were performed using Wilcoxon's rank sum test for continuous outcomes and the chi-squared test for dichotomous outcomes within each of the four strata. Changes in BMI-SDS during followup were also calculated via linear regression and adjusted for age, sex, migration background, and lifestyle intervention. Two-sided $p$ values were adjusted for multiple testing by Bonferroni stepdown method and $p<0.05$ was considered significant.

\section{Results}

A total of 76,660 children and adolescents met the inclusion criteria. The baseline characteristics are presented in Table 1 . The CVRFs are demonstrated in Figs. 1-4 stratified by age, sex, and respective definition of obesity classes.

\section{CVRFs in females $<10$ years}

BMI-SDS was related significantly $(p<0.001)$ to systolic blood pressure ( $\beta$-coefficient $4.0 \pm 0.3$ ) and diastolic blood pressure ( $\beta$-coefficient $2.2 \pm 0.3$ ). Systolic and diastolic blood pressures were significantly higher in class IV compared to class III obesity based on the 95th percentile BMI definition, while systolic and diastolic blood pressure did not differ significantly according to the IOTF definition.

Triglycerides, HDL cholesterol, and T/H correlated significantly $(p<0.001)$ with BMI-SDS $(\beta$-coefficients $10.1 \pm$
$2.1,-3.6 \pm 0.4,0.4 \pm 0.1$, respectively), but not LDL or non-HDL cholesterol. None of the lipids differed significantly between classes IV and III obesity regardless of the definition.

AST, ALT, and yGT correlated significantly $(p<0.005)$ with BMI-SDS ( $\beta$-coefficients $1.1 \pm 0.4,5.7 \pm 0.7,2.5 \pm 0.4$, respectively). None of the liver enzymes differed significantly between classes IV and III obesity regardless of the definition.

$\mathrm{HbA1c}$ and fasting glucose were not related to BMISDS, while 2-h glucose in oGTT was related $(p=0.001)$ to BMI-SDS ( $\beta$-coefficient $3.7 \pm 1.1$ ). HbA1c, fasting glucose, and 2-h glucose in oGTT did not differ significantly between classes III and IV obesity regardless of the definition.

\section{CVRFs in males $<12$ years}

BMI-SDS was related significantly $(p<0.001)$ to systolic blood pressure ( $\beta$-coefficient $4.5 \pm 0.3$ ) and diastolic blood pressure ( $\beta$-coefficient 2.6 0.2). Systolic and diastolic blood pressures were significantly higher in class IV compared to class III obesity based on the 95th percentile BMI definition, while systolic and diastolic blood pressure did not differ significantly according to the IOTF definition.

Triglycerides, HDL cholesterol, and T/H correlated significantly $(p<0.001)$ with BMI-SDS $(\beta$-coefficients $8.5 \pm$ $1.5,-4.8 \pm 0.3,0.4 \pm 0.0$, respectively), but not LDL or non-HDL cholesterol. HDL-cholesterol levels were significantly lower and $\mathrm{T} / \mathrm{H}$ were significantly higher in class IV compared to class III obesity based on the 95 th percentile BMI definition but not on the IOTF definition, while none of the other lipids differed significantly between classes IV and III regardless of the definition applied.

AST, ALT, and $\gamma$ GT correlated significantly $(p<0.001)$ with BMI-SDS ( $\beta$-coefficients $3.5 \pm 0.4,8.7 \pm 0.8,3.4 \pm 0.5$, respectively). All liver enzymes were significantly higher in class IV compared to class III obesity based on the 95th percentile BMI definition, while there were no significant differences based on the IOTF definition.

Fasting glucose and $\mathrm{HbA} 1$ were not related to BMI-SDS, while 2-h glucose in oGTT was related significantly to BMI-SDS ( $\beta$-coefficient $3.2 \pm 1.1$ ). However, their values did not differ significantly between classes III and IV obesity regardless of the definition.

\section{CVRFs in females 10 to $<14$ years}

BMI-SDS was related significantly $(p<0.001)$ to systolic blood pressure ( $\beta$-coefficient $8.4 \pm 0.2$ ) and diastolic blood pressure ( $\beta$-coefficient $4.7 \pm 0.2$ ). Systolic and diastolic blood pressure were significantly higher in class IV compared to class III obesity regardless of the definition. 


\section{IOTF definition}

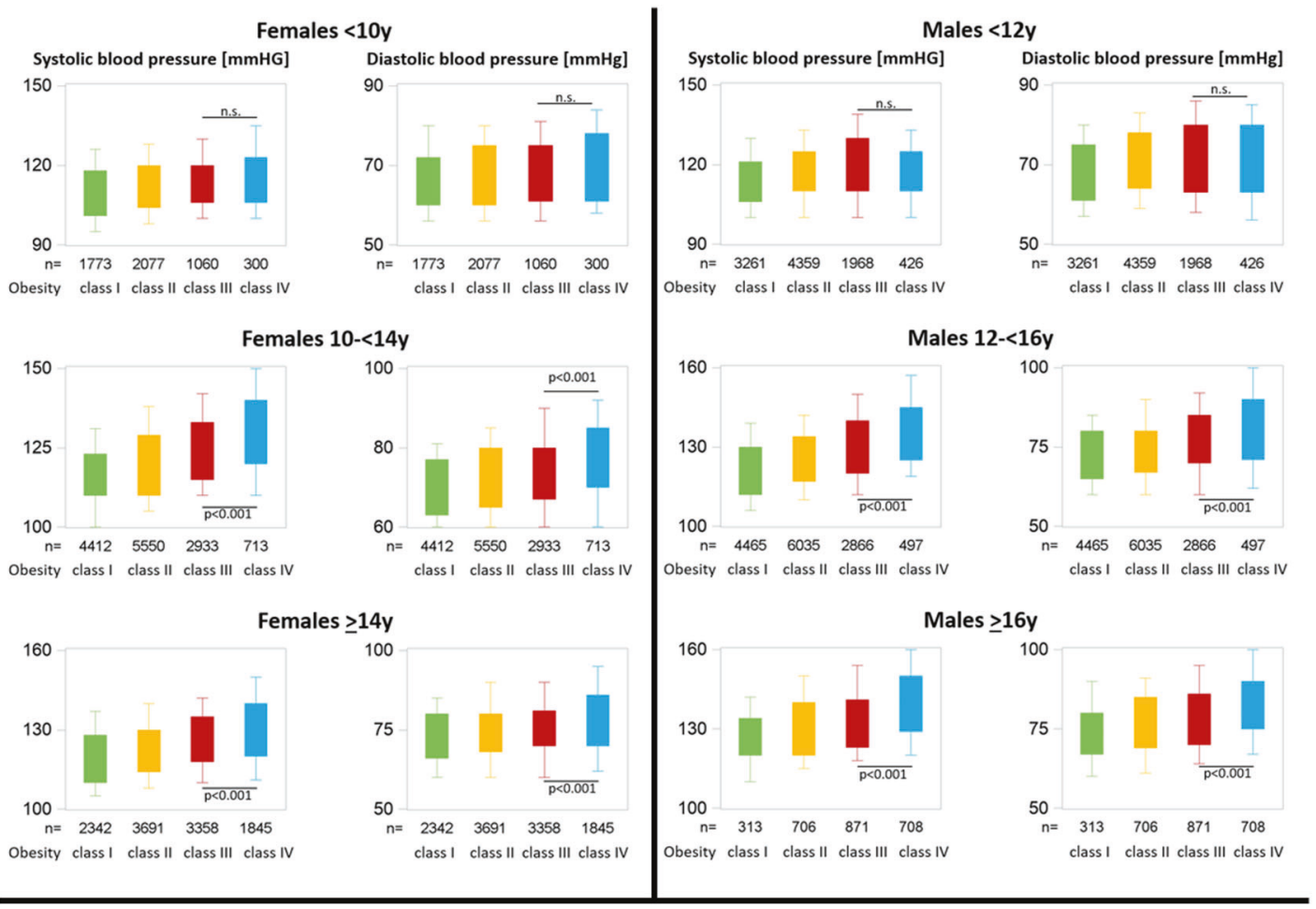

95th percentile BMI definition

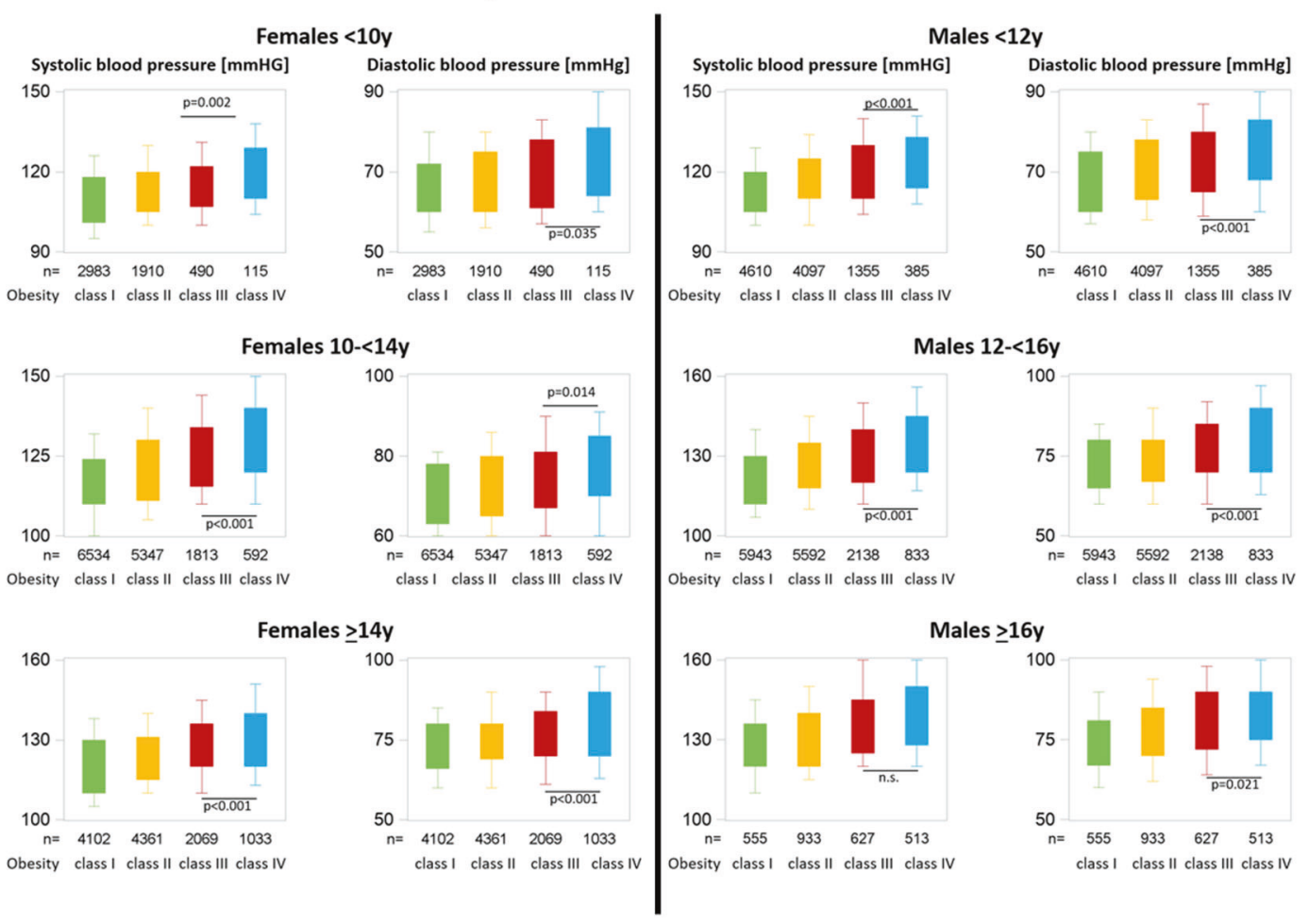

Fig. 1 Blood pressure stratified by obesity class, sex, and age using two different obesity class definitions. 


\section{IOTF definition}

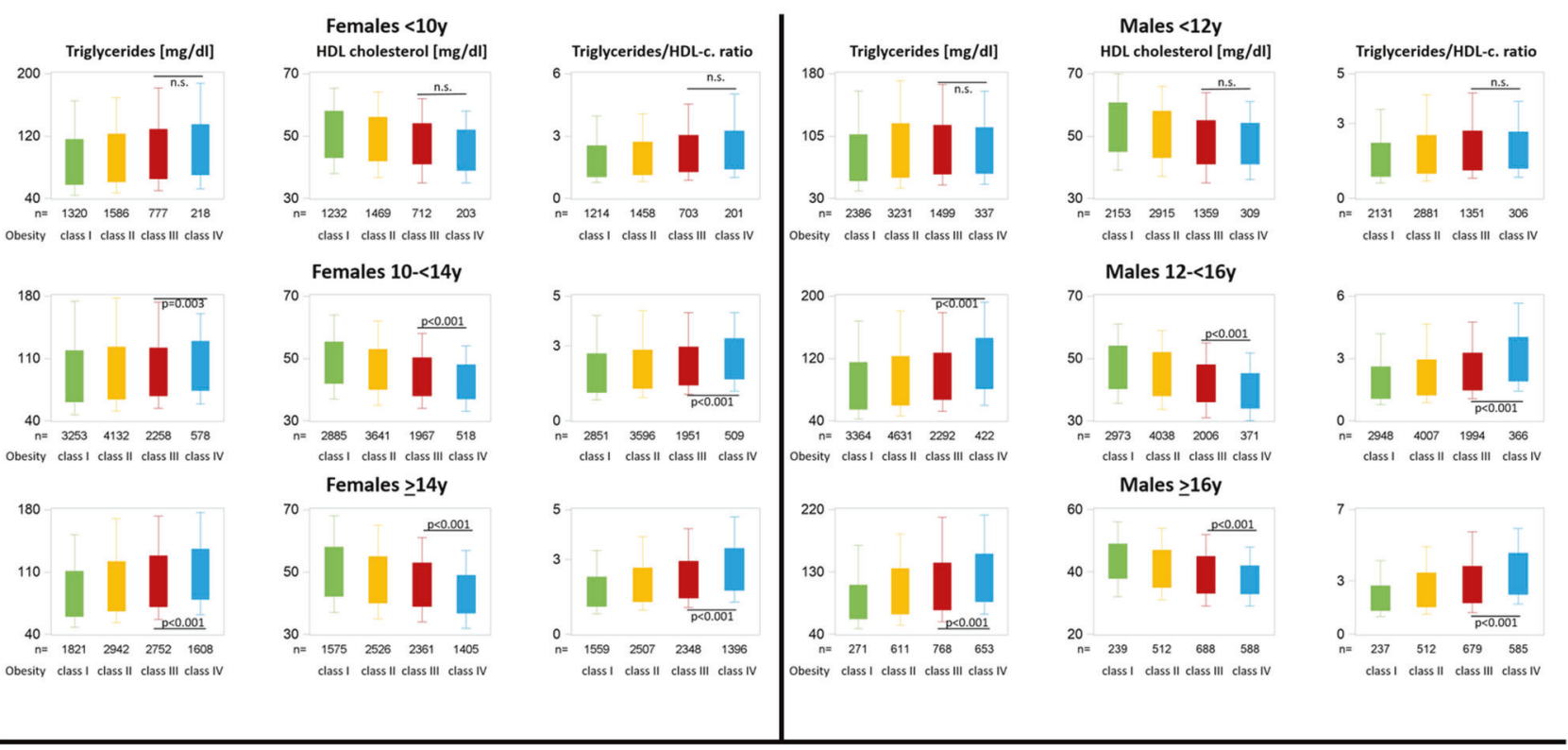

\section{5th percentile BMI definition}
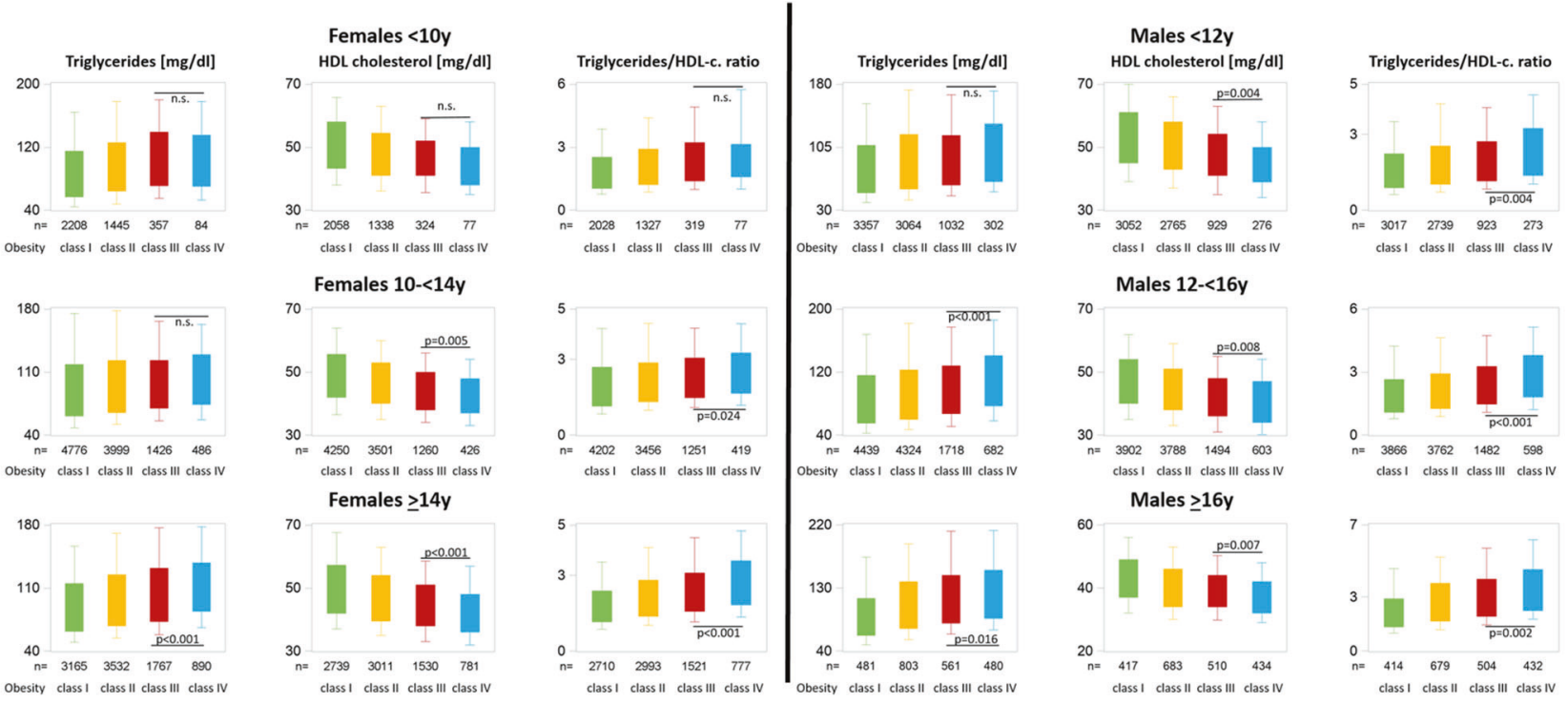

Fig. 2 Lipids stratified by obesity class, sex, and age using two different obesity class definitions.

Triglycerides, HDL cholesterol, LDL cholesterol, and T/ $\mathrm{H}$ correlated significantly $(p=0.002)$ with BMI-SDS ( $\beta$-coefficients $5.6 \pm 1.3,-2.0 \pm 0.6,-4.5 \pm 0.2,0.3 \pm 0.0$, respectively), but not non-HDL cholesterol. All lipids except LDL- and non-HDL cholesterol showed statistically significant differences between classes III and IV obesity according to the IOTF definition. Using the 95th percentile BMI definition, only HDL cholesterol and T/H ratio differed significantly between classes III and IV obesity.

AST, ALT, and $\gamma \mathrm{GT}$ correlated significantly $(p<$ 0.001 ) with BMI-SDS ( $\beta$-coefficients $2.1 \pm 0.3,6.9 \pm 0.5$,
$3.9 \pm 0.4$, respectively). The liver enzyme $\gamma \mathrm{GT}$ was significantly higher in class IV compared to class III obesity, while the transaminases did not differ significantly between classes IV and III obesity regardless of the definition.

Fasting glucose and 2-h glucose in oGTT were significantly related to BMI-SDS ( $\beta$-coefficient $1.2 \pm 0.5$ and $3.9 \pm 0.8$, respectively). HbA1c showed no association with BMI-SDS. Both glucose parameters as well as HbA1c did not differ significantly between classes IV and III obesity regardless of the definition. 


\section{IOTF definition}

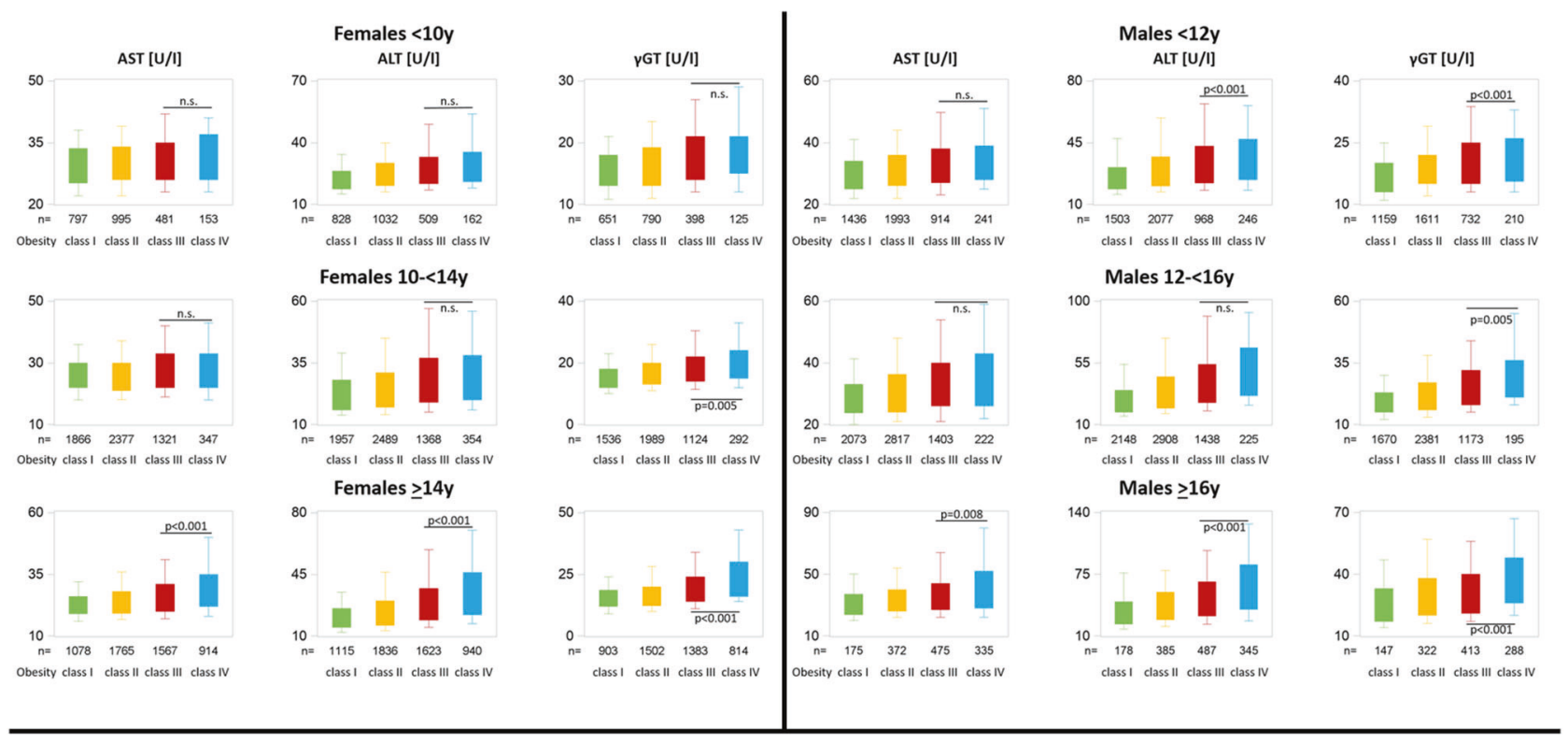

\section{5th percentile BMI definition}

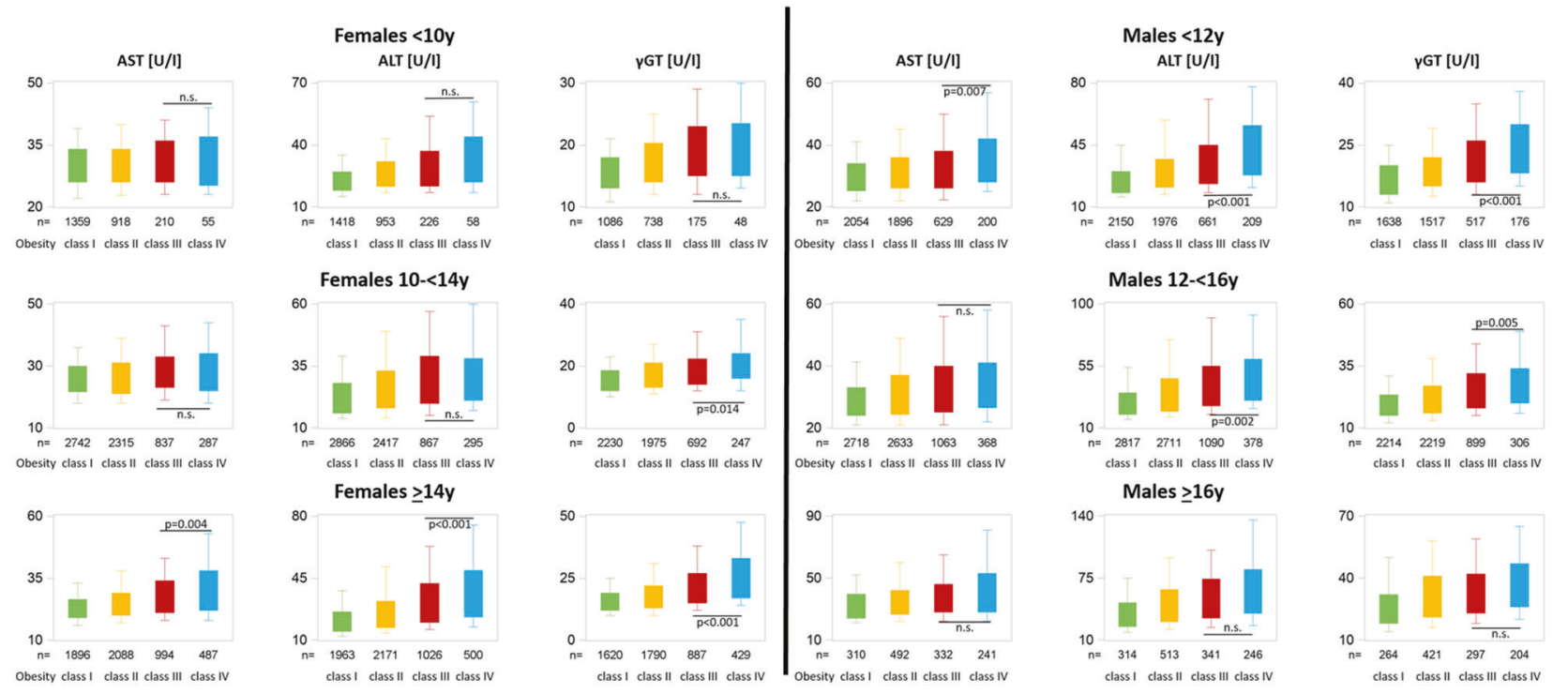

Fig. 3 Liver enzymes stratified by obesity class, sex, and age using two different obesity class definitions.

\section{CVRFs in males 12 to $<16$ years}

BMI-SDS was related significantly $(p<0.001)$ to systolic blood pressure ( $\beta$-coefficient $9.7 \pm 0.2)$ and diastolic blood pressure $(\beta$-coefficient $5.3 \pm 0.2$ ). Systolic and diastolic blood pressure levels were significantly higher in class IV compared to class III obesity regardless of the definition used.

Triglycerides, LDL, HDL, and non-HDL cholesterol, as well as $\mathrm{T} / \mathrm{H}$, correlated significantly $(p<0.001)$ with BMISDS ( $\beta$-coefficients $13.2 \pm 1.4,3.2 \pm 0.7,-5.6 \pm 0.3,4.3 \pm$
$0.9,0.6 \pm 0.0$, respectively). All lipids except LDL cholesterol showed statistically significant differences between classes III and IV obesity regardless of the definition.

AST, ALT, and $\gamma$ GT correlated significantly $(p<0.001)$ with BMI-SDS ( $\beta$-coefficients $6.1 \pm 0.4,15.0 \pm 0.8,7.2 \pm$ 0.4 , respectively). Levels of $\gamma$ GT were significantly higher in class IV compared to class III obesity, while AST did not differ significantly between classes IV and III regardless of the definition. ALT concentrations were significantly higher in class IV obesity compared to class III only if the 95th BMI percentile definition was applied. 


\section{IOTF definition}

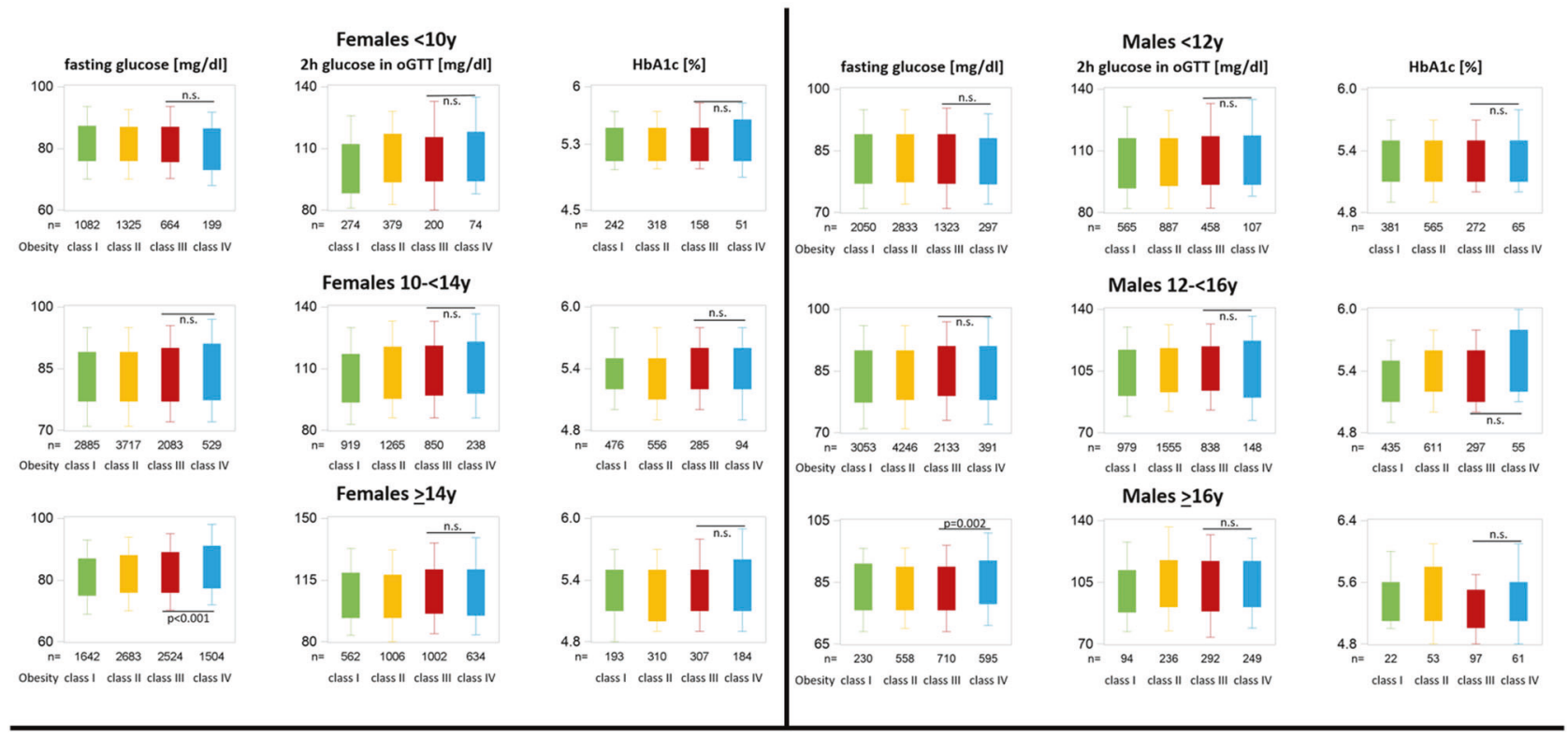

\section{5th percentile BMI definition}
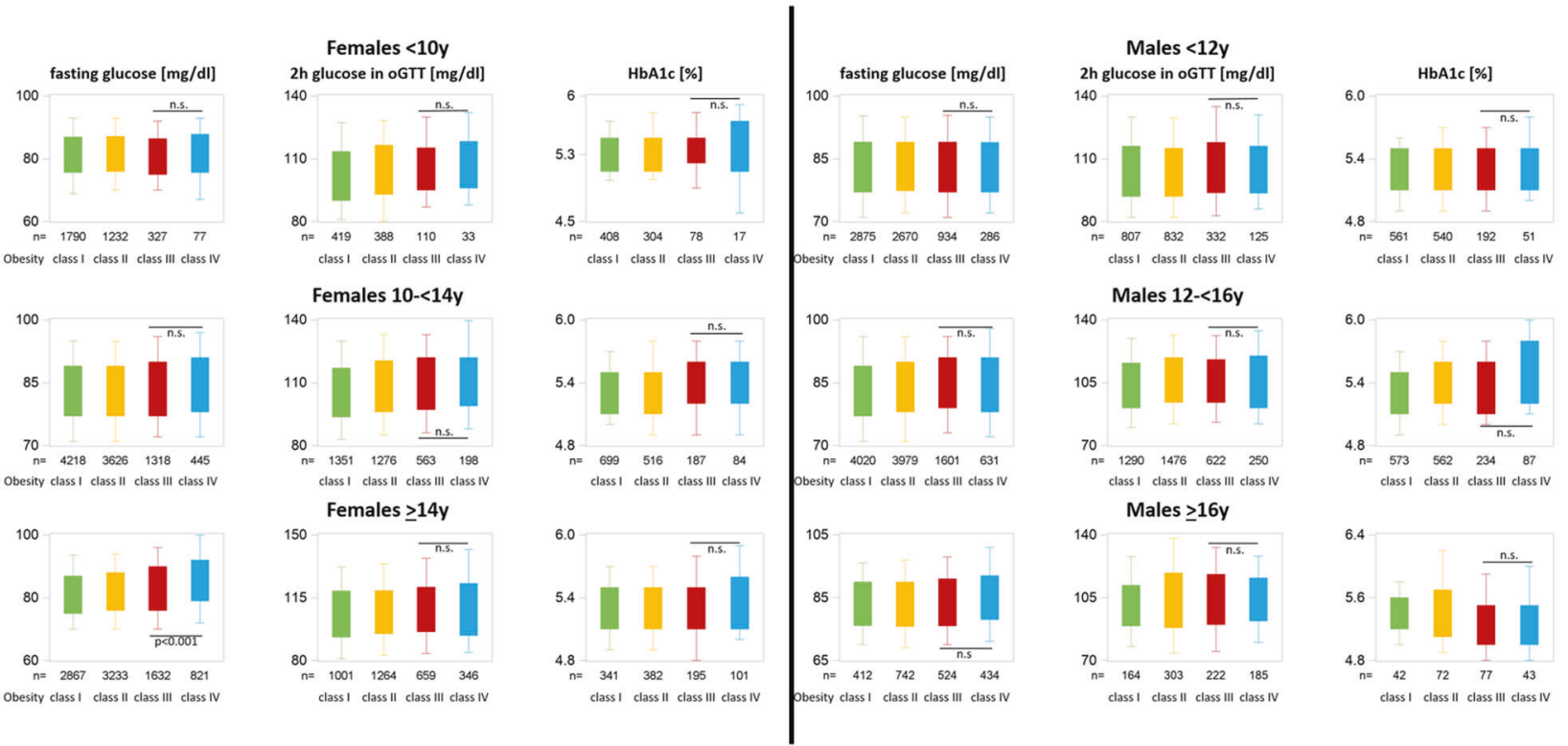

Fig. 4 Glucose metabolism parameters stratified by obesity class, sex, and age using two different obesity class definitions.

Fasting glucose, 2-h glucose in oGTT, and HbA1 correlated significantly $(p<0.05)$ with BMI-SDS ( $\beta$-coefficients $1.3 \pm 0.4,2.1 \pm 0.8,0.08 \pm 0.03$, respectively). Their values did not differ significantly between classes III and IV obesity regardless of the definition.

\section{CVRFs in females $>14$ years}

BMI-SDS was related significantly $(p<0.001)$ to systolic blood pressure ( $\beta$-coefficient $6.3 \pm 0.2$ ) and diastolic blood pressure (b-coefficient 3.6 0.2). Systolic and diastolic blood pressure values were significantly higher in class IV compared to class III obesity regardless of the definition used.

All lipid parameters were significantly $(p<0.001)$ related to degree of overweight ( $\beta$-coefficients triglycerides: $12.3 \pm$ 1.1, LDL cholesterol: $2.5 \pm 0.6$, HDL cholesterol: $-4.8 \pm$ 0.2 , non-HDL cholesterol: $4.3 \pm 0.7, \mathrm{~T} / \mathrm{H}$ ratio: $0.5 \pm 0.0$, respectively). All lipids except LDL cholesterol and nonHDL cholesterol showed statistically significant differences 
between classes III and IV obesity regardless of the definition.

AST, ALT, and $\gamma$ GT correlated significantly $(p<0.001)$ with BMI-SDS ( $\beta$-coefficients $4.6 \pm 0.3,9.9 \pm 0.6,5.7 \pm 0.4$, respectively). All liver enzymes were significantly higher in class IV compared to class III obesity regardless of the definition.

Fasting glucose and 2-h glucose in oGTT were significantly related to BMI-SDS ( $\beta$-coefficient $2.9 \pm 0.5$ and $3.2 \pm 0.7$, respectively), but only fasting glucose was significantly higher in class IV compared to class III obesity regardless of the definition. HbAlc showed no association with BMI-SDS.

\section{CVRFs in males $>16$ years}

BMI-SDS was related significantly $(p<0.001)$ to systolic blood pressure ( $\beta$-coefficient $7.4 \pm 0.5$ ) and diastolic blood pressure ( $\beta$-coefficient $5.6 \pm 0.4$ ). Systolic and diastolic blood pressure levels were significantly higher in class IV compared to class III obesity using the IOTF definition, in the 95th percentile BMI definition only diastolic blood pressure was significantly higher.

All lipid parameters were significantly related to degree of overweight ( $\beta$-coefficients triglycerides: $19.4 \pm 2.5$, LDL cholesterol: $7.3 \pm 1.3$, HDL cholesterol: $-3.6 \pm 0.4$, nonHDL cholesterol: $8.3 \pm 1.6, \mathrm{~T} / \mathrm{H}$ ratio: $0.7 \pm 0.1$, respectively). Triglycerides, HDL cholesterol, and the T/H ratio showed statistically significant differences between classes III and IV obesity under both classifications, while LDL and non-HDL cholesterol differed significantly only using the IOTF definition.

AST, ALT, and $\gamma$ GT correlated significantly $(p<0.001)$ with BMI-SDS ( $\beta$-coefficients $7.1 \pm 1.1,18.3 \pm 1.8,7.1 \pm$ 1.3 , respectively). All liver enzymes differed significantly between classes III and IV obesity using the IOTF definition, while using the 95th percentile BMI definition no significant differences were revealed.

Fasting glucose, 2-h glucose in oGTT, and HbA1 were not related to BMI-SDS. Only fasting glucose was significantly higher in class IV compared with class III using the IOTF definition.

\section{Prevalences of hypertension, dyslipidemia, and T2DM}

The prevalences of hypertension, dyslipidemia, and T2DM are given in Fig. 5 stratified by age, sex, and obesity classes. Hypertension was highly prevalent and significantly more frequent in class IV compared to class III obesity regardless of the applied definition in boys 12 to $<16$ years and girls $\geq$ 10 years. In girls $<10$ years, prevalence of hypertension was significantly higher in class IV compared to class III obesity only using the 95th percentile BMI definition. In boys $\geq 16$ years, prevalence of hypertension was significantly higher in class IV compared to class III obesity only when the IOTF definition was used.

Prevalence of dyslipidemia was also considerable but did not differ significantly between classes III and IV obesity in girls $<14$ years and boys $<12$ years independent of the definition used.

Prevalence of T2DM was generally very low and did not differ significantly between children and adolescents with class IV compared to class III obesity regardless of the definition, age, or sex except females $\geq 14$ years. Those girls showed a higher prevalence of T2DM in class IV compared to class III obesity but only when the IOTF definition was used.

\section{Longitudinal analyses}

A total of 1089 children and adolescents with bariatric surgery or with long-term inpatient treatment $>6$ months were excluded from the longitudinal analyses. Total number of children included and their respective BMI-SDS with available follow-up are presented in Supplementary Table 1.

Due to the too small percentages of patients with available follow-up (maximum $34 \%$ and mean $<20 \%$ at 1 year, maximum $19 \%$ and mean $<12 \%$ at 2 years), we did not analyze the impact of different obesity classes on CVRF longitudinally. The numbers of children with available follow-up decreased with observation period and age at baseline in all six strata.

BMI-SDS reduced significantly $(<0.001)$ in follow-up at 1 and 2 years. The BMI-SDS reduction was not significant different between classes III and IV obesity regardless of the underlying definition, age, or sex except the two subgroups females $<10$ years and boys $<12$ years: these two groups showed a significantly ( $p<0.05$, respectively) larger decrease in BMI-SDS over 1 and 2 years in the class IV compared to class III obesity according to the IOTF definition but not according to the 95th percentile definition.

\section{Discussion}

To the best of our knowledge, this is the largest study of children and adolescents with classes III and IV obesity presenting at tertiary obesity treatment centers in Central Europe. In line with our hypothesis, many CVRFs did not differ significantly between children with classes III and IV obesity, while there were significant differences in some CVRFs between class III and IV obesity in adolescents.

The relationship between most analyzed CVRFs and classes of obesity was very similar in the IOTF and percentage of 95th percentile of BMI definition. However, in 

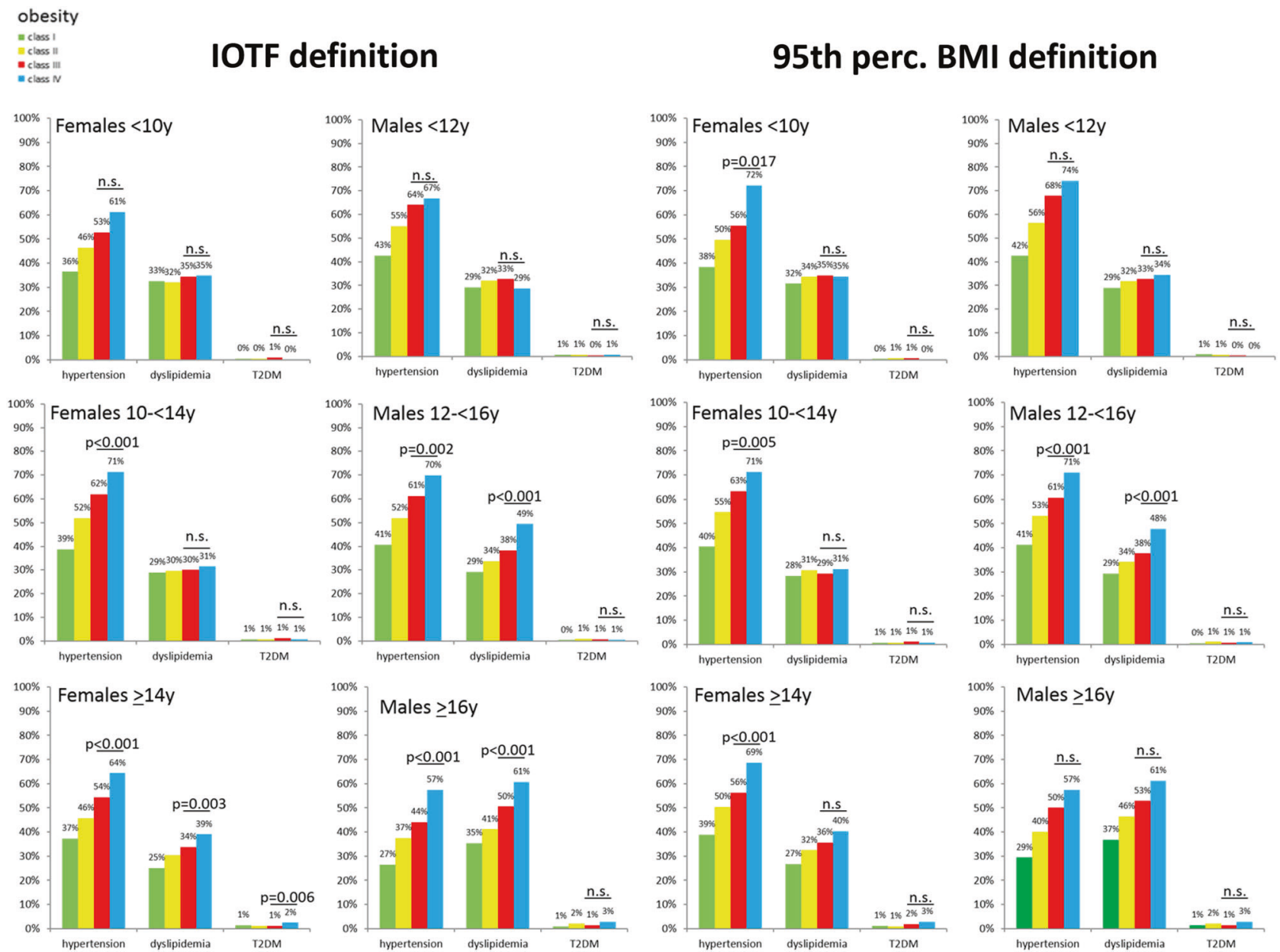

Fig. 5 Prevalences of type 2 diabetes mellitus (T2DM), dyslipidemia, and hypertension stratified by age group, sex, and obesity class using two different obesity class definitions.

children more significant differences between classes III and IV obesity in respect to CVRFs were found using the 95th BMI percentile definition, while in adolescents the opposite was observed. However, the pattern of significant differences between classes IV and III obesity is difficult to interpret in favor of one of the two classifications, since statistical significance is dependent on effect size and sample size. In the IOTF definition more children and adolescents are classified as class IV or III obese than in the definition based on the percentage of 95th percentile of BMI. This is also reflected in higher BMI values.

We found a linear relationship between degree of overweight expressed as BMI-SDS and systolic and diastolic blood pressure (positive), triglycerides (positive), HDL cholesterol (negative), liver enzymes (positive), and the T/H ratio (positive) as indirect parameter of insulin resistance at any age and sex group. Fasting glucose and 2-h glucose in oGTT correlated significantly to BMI-SDS in some subgroups (e.g., females $>14$ years and males $12-16$ years). These findings are in line with the study of Skinner et al. highlighting the increased metabolic risk of rising degrees of obesity in childhood [4]. Of interest all these CVRFs are related to insulin resistance [5-8].

However, many of these associations got lost when comparing obesity classes III and IV in different age groups. In prepubertal children (females $<10$ years and males $<12$ years), only blood pressure levels in both sexes and HDL cholesterol concentrations in boys differed significantly between classes IV and III obesity. Furthermore, this was only true using the 95th percentile BMI definition but not if applying the IOTF definition. Additionally, all other CVRFs did not differ significantly in those children. In pubertal and post pubertal age (females $>10$ years and males $\geq 12$ years), most CVRFs differed significantly between classes III and IV obesity. However, glucose parameters in any age and sex group (except fasting glucose in females $>14$ years) and transaminases in females aged $10-14$ years did not differ significantly. Females $\geq 10$ years and males $\geq 12$ to $<16$ years with class IV obesity had higher prevalences of hypertension than adolescents with 
class III obesity independent of the applied obesity class definition, while prevalence of dyslipidemia did not differ significantly between classes IV and III obesity except females $\geq 14$ years (only if using the IOTF definition). In males $\geq 16$ years, a significant higher prevalence of hypertension was only observed applying the IOTF definition. Independent of age, sex, and used obesity class definition, the prevalences of T2DM did not differ between classes III and IV obesity (except females $\geq 14$ years). These findings are in concordance with the hypothesis of Zabarsky et al. questioning a linear relationship between CVRFs and degree of overweight in the upper BMI cut-offs [10]. Besides age and sex, CVRFs are dependent on additional factors such as genetic background, physical fitness, or dietary components apart from degree of overweight. Genetic correlations between childhood obesity versus adult BMI and fasting glucose or T2DM have been shown to be lower, thus supporting the notion that genetic factors contributing to a high BMI in childhood do not substantially overlap with those for the respective parameters of glucose metabolism [22]. Potentially, this low overlap partially explains our nonsignificant correlations between obesity and parameters of glucose metabolism.

Categorization of degrees of obesity according to BMI thresholds is an easy tool. However, the important limitation of this approach is that other important factors influencing CVRFs such as, for example, body fat distribution are neglected [23]. Furthermore, it has been shown in large cohort of Israeli adolescents that also a BMI above the 50th but below the 85 th percentile at age of 17 years was associated with cardiovascular morbidity and mortality in the next 40 years [3] demonstrating the limitation of the use of BMI cut-offs. Our analysis emphasizes that categorization by BMI thresholds has shortcomings for distribution of glucose metabolism at any age and for all other CVRFs except blood pressure especially in prepubertal children.

Of note, fasting glucose was related to BMI-SDS in females $\geq 14$ years and fasting glucose was significantly higher in females $\geq 14$ years with class IV compared to females $\geq 14$ years with class III obesity, while in males of any age and in females $<14$ years no significant association between BMI-SDS and fasting glucose was observed. This finding is in line with the observation of a female predominance in adolescents with T2DM not only in our study (1.3 versus $1.2 \% \mathrm{~T} 2 \mathrm{DM}$ in adolescents $\geq 14$ years, but no difference in children $<14$ years) but also within previous reports [24]. This suggests that besides a higher insulin resistance in girls compared to boys [25], the combination of obesity and female sex hormones or associated factors may support the development of T2DM or that male sex hormones or associated factors may prevent the development of T2DM, even if the effect seems to be small. Future studies are necessary to prove these hypotheses.
The vast majority of children attending a specialized treatment center of obesity were lost to follow-up thus casting doubt on the achievement of major improvement in their degree of overweight during the follow-up period. Furthermore, although the children and adolescents with follow-up data achieved a significant decrease of their degree of overweight, this decrease was only marginal and likely not relevant for the CVRFs since the decrease of BMI-SDS was $<0.2$ on average. It has been shown that a decrease of $>0.25$ BMI-SDS is necessary to improve CVRFs in children and adolescents [26]. This emphasizes the limitations of short-term inpatient or long-term outpatient standard-of-care conservative obesity management. We found no difference between classes III and IV obesity concerning change of BMI-SDS in females $\geq 10$ years and boys $>12$ years but a larger degree of BMI-SDS reduction in younger children with class IV obesity compared to class III obesity. This finding is in line with previous studies reporting that especially adolescents with class III or IV obesity in contrast to children with class III or IV obesity do not respond to lifestyle interventions [27, 28].

Almost 10 years ago, we reported that the vast majority of specialized treatment centers for children and adolescents with obesity participating in the APV quality control program were not able to prove the effectiveness of their lifestyle interventions under real-life conditions due to the high dropout rate of $>93 \%$ after 2 years [29]. Compared to these historical data, the drop-out rate in our recent analyses decreased probably due to the APV benchmarking process improving the quality of interventions. However, the drop-out rate is still too high to prove effectiveness. This finding highlights that achieving treatment adherence is the greatest challenge in treating children and adolescents with obesity. The causes of nonadherence have to be analyzed in order to judge if it is based on characteristics of patients with obesity (e.g., difficulties to visit the treatment centers regularly) or the interventions itself (e.g., not tailored specific underfinanced intervention with insufficient patient contacts). The problem of nonadherence has to be solved before the analysis of effectiveness of treatment strategies such as bariatric surgery, medications, or more intensified lifestyle interventions.

The strengths of our study are the very large study sample, the multicenter design, and the analysis under reallife conditions and not under ideal circumstances of study trials with selected patients. However, we have to mention some important limitations of the study. First, not all obesity treatment centers in the analyzed region participated in this study. Nevertheless, the most specialized treatment centers are likely to be enrolled in this study at least in Germany, since participating in the APV quality program is necessary for funding of intervention by German health insurances and accreditation of obesity treatment centers. Second, our reference group of subjects with overweight or 
obesity was referred to specialized obesity clinics. Therefore, they may be characterized by a more pronounces CVRF profile compared to subjects with similar anthropometric measures derived from the population. Third, we do not have complete data on pubertal stages. Therefore, the three age categories used in this study are only an approximation of pubertal stage. Fourth, we performed no study visits to monitor for correct measurement, for example, of blood pressure. However, data were determined by the obesity specialist centers according to German guidelines. Finally, the drop-out rate at follow-up was too high to calculate longitudinal relationships between CVRFs and changes of weight status.

In summary, we demonstrated the great challenge of treatment adherence in children and adolescents with obesity. We showed that prevalences of hypertension and dyslipidemia did not differ significantly or consistently between classes III and IV obesity in males $\leq 12$ years and females $\leq 10$ years. Disturbances of glucose metabolism including T2DM did not differ significantly between classes III and IV obesity in boys of any age or in girls $<14$ years. Therefore, the presence of CVRFs and not the BMI cut-offs should primarily guide treatment decisions such as medications or bariatric surgery. More longitudinal studies with high follow-up rate are needed to justify decisions being based solely on obesity grades.

Acknowledgements The APV standardized documentation was supported by grants of the German "Competence Network Obesity," which is supported by the German Federal Ministry of Education and Research (Grant Number 01 GI0839), in part as funding for the YESstudy, and by grants of the German Obesity Society (DAG). We are indebted to all health professionals of the APV Study Group taking care of overweight children and contributing to the APV database.

Funding The work described in this paper was supported by the Innovative Medicines Initiative of the European Union (No. 875534SOPHIA). Open Access funding enabled and organized by Projekt DEAL.

\section{Compliance with ethical standards}

Conflict of interest The authors declare no competing interests.

Publisher's note Springer Nature remains neutral with regard to jurisdictional claims in published maps and institutional affiliations.

Open Access This article is licensed under a Creative Commons Attribution 4.0 International License, which permits use, sharing, adaptation, distribution and reproduction in any medium or format, as long as you give appropriate credit to the original author(s) and the source, provide a link to the Creative Commons license, and indicate if changes were made. The images or other third party material in this article are included in the article's Creative Commons license, unless indicated otherwise in a credit line to the material. If material is not included in the article's Creative Commons license and your intended use is not permitted by statutory regulation or exceeds the permitted use, you will need to obtain permission directly from the copyright holder. To view a copy of this license, visit http://creativecommons. org/licenses/by/4.0/.

\section{References}

1. Han JC, Lawlor DA, Kimm SY. Childhood obesity. Lancet. 2010;375:1737-48.

2. Juonala M, Magnussen CG, Berenson GS, Venn A, Burns TL, Sabin M. et al. Childhood adiposity, adult adiposity, and cardiovascular risk factors. N Engl J Med. 2011;365:1876-85.

3. Twig G, Yaniv G, Levine H, Leiba A, Goldberger N, Derazne E, et al. Body-mass index in 2.3 million adolescents and cardiovascular death in adulthood. N Engl J Med. 2016;374:2430-40.

4. Skinner AC, Perrin EM, Moss LA, Skelton JA. Cardiometabolic risks and severity of obesity in children and young adults. N Engl J Med. 2015;373:1307-17.

5. Chu NF, Rimm EB, Wang DJ, Liou HS, Shieh SM. Clustering of cardiovascular disease risk factors among obese schoolchildren: the Taipei Children Heart Study. Am J Clin Nutr. 1998;67:1141-6.

6. Csabi G, Torok K, Jeges S, Molnar D. Presence of metabolic cardiovascular syndrome in obese children. Eur $\mathrm{J}$ Pediatr. 2000;159:91-4.

7. Freedman DS, Dietz WH, Srinivasan SR, Berenson GS. The relation of overweight to cardiovascular risk factors among children and adolescents: the Bogalusa Heart Study. Pediatrics. 1999;103:1175-82.

8. I'Allemand D, Wiegand S, Reinehr T, Müller J, Wabitsch M, Widhalm K, et al. Cardiovascular risk in 26,008 European overweight children as established by a multicenter database. Obesity. 2008;16:1672-9.

9. Reinehr T, Wolters B, Knop C, Lass N, Holl RW. Strong effect of pubertal status on metabolic health in obese children: a longitudinal study. J Clin Endocrinol Metab. 2015;100:301-8.

10. Zabarsky G, Beek C, Hagman E, Pierpont B, Caprio S, Weiss R. Impact of severe obesity on cardiovascular risk factors in youth. J Pediatr. 2018;192:105-14.

11. Fried M, Yumuk V, Oppert JM, Torres A, Weiner R, Yashkov Y, et al. Interdisciplinary European guidelines on metabolic and bariatric surgery. Obes Surg. 2014;24:42-55.

12. Styne DM, Arslanian SA, Connor EL, Farooqi IS, Murad MH, Silverstein JH, et al. Pediatric obesity-assessment, treatment, and prevention: an endocrine society clinical practice guideline. J Clin Endocrinol Metab. 2017;102:709-57.

13. German Obesity Association. Guidelines of the German working group on obese children and adolescents. www.a-g-a.de/Leitlinie. pdf. assessed Jan 2020.

14. Reinehr T, Wabitsch M, Andler W, Beyer P, Böttner A, ChenStute A, et al. Medical care of obese children and adolescents. APV: a standardised multicentre documentation derived to study initial presentation and cardiovascular risk factors in patients transferred to specialised treatment institutions. Eur J Pediatr. 2004;163:308-12.

15. Cole TJ, Lobstein T. Extended international (IOTF) body mass index cut-offs for thinness, overweight and obesity. Pediatr Obes. 2012;7:284-94.

16. Cole TJ. The LMS method for constructing normalized growth standards. Eur J Clin Nutr. 1990;44:45-60.

17. Rosenbauer J, Dost A, Karges B, Hungele A, Stahl A, Bächle C, et al. Improved metabolic control in children and adolescents with type 1 diabetes: a trend analysis using prospective multicenter data from Germany and Austria. Diabetes Care. 2012;35:80-6.

18. Zeitler P, Arslanian S, Fu J, Pinhas-Hamiel O, Reinehr T, Tandon N, et al. ISPAD Clinical Practice Consensus Guidelines 2018: type 2 diabetes mellitus in youth. Pediatr Diabetes. 2018;19 Suppl 27:28-46.. 
19. Guidelines of the German paediatric working group on metabolic disorders. https://www.awmf.org/leitlinien/detail/11/027-068.html. assessed Jan 2020.

20. Rao G. Diagnosis, epidemiology, and management of hypertension in children. Pediatrics. 2016;138:e20153616.

21. Neuhauser HK, Thamm M, Ellert U, Hense HW, Rosario AS. Blood pressure percentiles by age and height from nonoverweight children and adolescents in Germany. Pediatrics. 2011;127: e978-88.

22. Bulik-Sullivan B, Finucane HK, Anttila V, Gusev A, Day FR, Loh PR. An atlas of genetic correlations across human diseases and traits. Nat Genet. 2015;47:1236-41.

23. Weiss R. Fat distribution and storage: how much, where, and how? Eur J Endocrinol. 2007;157 Suppl 1:S39-45.

24. Klingensmith GJ, Lanzinger S, Tamborlane WV, Hofer SE, Cheng P, de Beaufort C. et al. Adolescent type 2 diabetes: comparing the Pediatric Diabetes Consortium and Germany/Austria/ Luxemburg Pediatric Diabetes Prospective registries. Pediatr Diabetes. 2018;19:1156-63.
25. Allard P, Delvin EE, Paradis G, Hanley JA, O'Loughlin J, Lavallée C. et al. Distribution of fasting plasma insulin, free fatty acids, and glucose concentrations and of homeostasis model assessment of insulin resistance in a representative sample of Quebec children and adolescents. Clin Chem. 2003;49:644-9.

26. Reinehr T, Lass N, Toschke C, Rothermel J, Lanzinger S, Holl $\mathrm{RW}$. Which amount of BMI-SDS reduction is necessary to improve cardiovascular risk factors in overweight children? J Clin Endocrinol Metab. 2016;101:3171-9.

27. Danielsson P, Kowalski J, Ekblom O, Marcus C. Response of severely obese children and adolescents to behavioral treatment. Arch Pediatr Adolesc Med. 2012;166:1103-8.

28. Knop C, Singer V, Uysal Y, Schaefer A, Wolters B, Reinehr T. Extremely obese children respond better than extremely obese adolescents to lifestyle interventions. Pediatr Obes. 2015;10:7-15.

29. Reinehr T, Widhalm K, l'Allemand D, Wiegand S, Wabitsch M, Holl RW. Two-year follow-up in 21,784 overweight children and adolescents with lifestyle intervention. Obesity. 2009; 17:1196-9. 